initially positive and proportional to the applied stress. The ratio between these two quantities, which is known as the 'stress-optical coefficient', may be seen from curve $(a)$ in the accompanying graph to be about 10 brewsters. If stresses above about $10^{8}$ dynes/cm. ${ }^{2}$ are maintained, the birefringence decreases with time, and if the stress is then removed a negative birefringence results. This residual birefringence then relaxes slowly. For pressures greater than $8 \times 10^{8}$ dynes $/ \mathrm{cm}^{2}$, the birefringence is found to pass through zero and become negative even when the stress is maintained.

The graph shows the results obtained at $16^{\circ} \mathrm{C}$. with monoehromatic light $(\lambda=5461 \mathrm{~A}$.) for polystyrene under compression when the stresses have been maintrined for various times. These results appear to indicate that two distinct molecular mechanisms take part in the mechanical deformation of polystyrene at room temperature. On first applying a stress, sections of the molecular chains tend to become aligned, and this is associated with the elastic behaviour of the material. When the stress is maintained, however, an orientation of the benzene groups slowly occurs, and this 'flow' takes a corresponding time to relax when the stress is removed. It is hoped to publish fuller results elsewhere of the behaviour at different temperatures.

Imporial Chemical Industries, Ltd.,

H. KoLSKY

Butterwick Research Laboratories,

The Frythe, Welwyn, Herts. April 4.

' Kolsky, H., and Shearman, A. C., Proc. Phys. Soc., 55, 383 (1943). ${ }^{2}$ Nielsen, I. E., and Buchdah], K., J. Chem. Phys., 17, 839 (1949).

${ }^{3}$ Signer, R., Trans. Farad. Soc., 32, 296 (1936).

\section{Interlamellar Adsorption of Protein Monolayers on Pure Montmorillonoid
Cláys}

ORIENTED fiakes of the fine fractions (less than $0.5 \mu$ ) of montmorillonite and hectorite, when dipped in protein suspensions at $p H$ values far below their iso-electric point, exhibit a behaviour similar in general to that observed by Gieseking and his collaborators ${ }^{1}$. The difference in technique, however, shows a striking modification in the resulting proteinclay complexes. After immersion in gelatin-suspensions of $p H 2.5$ and concentrations stronger than $I$ per cent, unidimensional Fourier syntheses of the X-ray diagrams of the oriented flake suggest that : (a) two monolayers, $4 \cdot 3 \mathrm{~A}$. thick, of the polypeptide chains of the protein are taken up in the interlamellar space, and completely fill it; $(b)$ each monolayer is strongly adsorbed on the clay mineral sheets, and a high degree of order along the $c$-axis is obtained in the sandwich thus formed. The X-ray diagram is analogous to that of the glycerol-montmorillonite complex. This lends strong support to the idea that surface adsorptive forces play a major part in the formation of these two-layer organic complexes in montmorillonoid clays.

It is also possible to obtain what appears to be a one-layer $(4 \cdot 8 \mathrm{~A}$. thick) complex of gelatin with a $c$-spacing of $14 \cdot 2 \mathrm{~A}$. by two alternative methods: (i) by dipping the oriented clay flake in gelatin suspensions at concentrations 0.5 per cent and less; (ii) by dipping a flake of the two-layer complex in a 1 per cent gelatin suspension at $p \cdot \mathbf{H}$ values greater than $3 \cdot 5$. The $\mathrm{X}$-ray diagrams of these flakes suggest that either $(a)$ the monolayer taken up does not fill the interlamellar space completely, or (b) that the monolayer lies in the interlamellar space in a crinkled state, thus preventing any high degree of order in the $c$-dimension.

It is possible to pass from the one-layer complex into the highly ordered two-layer complex by dipping a flake of the former in a 1 per cent gelatin suspension at $p \mathrm{H} 2 \cdot 5$. Under exceptional conditions, which are difficult to reproduce always (that is, with flakes formed by very slow deposition of the clay particles of less than $0 \cdot 1 \mu$ diameter), a complex with a $c$ spacing of $26.3 \mathrm{~A}$. is formed (in addition to the twolayer complex) which appears to be a four-layer complex. Like the one-layer complex, the four-layer complex does not show such a high degree of order in the c-dimension as is observed in the two-layer complex.

Identical results for the one- and two-layer complexes are obtained with edestin and pepsin suspensions. Flakes dipped in 1 per cent solutions of the amino-acids glycine and arginine at $p \mathbf{H} 2.5$ give X-ray diagrams similar to that of the one-layer protein complex, except that the increment in the $c$-spacing is $3.5 \mathrm{~A}$. In all cases the flakes are dried over phosphorus pentoxide in an evacuated desiccator overnight before X-ray examination.

The energy of adsorption between the clay sheets and the protein chains is primarily due to electro. static forces reinforced by strong Van der Waals' attraction. This is sufficient to cause protein molecules in suspension to uncoil into polypeptide chains, which then easily penetrate the interlamellar space. The two-layer complexes are the most stable of the three kinds observed, each mono-layer being firmly attached to opposite clay surfaces. The thickness of the monolayer observed $(4 \cdot 3 \mathrm{~A}$.) shows that sidechains must lie almost flat on the clay surfaces in the interlamellar space. This is not only in good agreement with the calculated and observed dimensions of polypeptide chains (from X-ray evidence) but also shows a remarkable similarity to 'State III' of the gel films of protein monolayers observed by Rideal and collaborators ${ }^{2}$ on an aqueous base, although the orientation of side-chains with respect to the. surface of the adsorbent is not the same.

The two-layer complex has some other significant characteristies. There is no uptake of glycerol or water after soaking the flakes in either liquid for more than forty-eight hours in the cold. This hydrophobicity is similar to that of amine-clay complexes described by Jordan ${ }^{3}$. However, the conversion of a compound, strongly hydrophilic in the natural state, into a hydrophobic complex by interlamellar adsorption is remarkable. Prolonged contact with $N$ hydrochloric acid has no effect on the complex; with $0.2 N$ sodium hydroxide, the complex shows signs of being attacked slowly after forty-eight hours contact.

Acknowledgments are due to the Department of Scientific and Industrial Research for a grant; and to Dr. D. M. C. MacEwan for originally suggesting the problem on the lines of Gieseking's work.

Pedology Department, O. TAlibUdenn

Rothamsted Experimental Station, Harpenden, Herts. March 4.

${ }^{1}$ Gieseking, Soil Sci., 48, 467 (1939).

2 Hughes and Rideal, Proc. Roy. Soc., A, 137, 62 (1932),

s Jordan, Min. Mag., 28, 598 (1949). 